\title{
Two-Step PCR in the Evaluation of Antibiotic Treatment for Ehrlichia platys Infection
}

\author{
Wen-Lan CHANG, Whey-Li SU1), and Ming-Jeng PAN* \\ Graduate Institute of Veterinary Medicine and ${ }^{1)}$ Veterinary Hospital, College of Agriculture, National Taiwan University, Taipei, \\ Taiwan, ROC \\ (Received 21 March 1997/Accepted 20 May 1997)
}

ABSTRACT. We evaluated the feasibility of using the two-step polymerase chain reaction (PCR) in determining the withdrawal time of antibiotic treatment for Ehrlichia platys infection. We also present experimental evidence of a dog remaining a carrier after treatment with tetracycline. Canine infectious cyclic thrombocytopenia (CICT) was induced in 3 dogs by intravenous inoculation of blood infected with E. platys. Tetracycline was administered to one of the dogs for 2 weeks when parasitemia appeared. Although the hematologic abnormality of cyclic thrombocytopenia soon disappeared, a few parasitized platelets reappeared after the withdrawal of treatment, and the dog thus remained as a carrier. The other dogs were treated with doxycycline when parasitemic episodes first developed. The durations of antibiotic regimens were determined by the results of two-step PCR in which the 16S rDNA of E. platys was amplified from blood samples. Doxycycline was withdrawn after 8 days of treatment, and the follow-up monitoring continued for 3 weeks. The platelet counts of the 2 dogs remained within the normal range, and the etiologic agent of CICT was not found either by Giemsa staining or by the twostep PCR, indicating complete elimination of the agent. - KEY wORDS: canine infectious cyclic thrombocytopenia, Ehrlichia platys, polymerase chain reaction.

J. Vet. Med.Sci. 59(9): 849-851, 1997

Four species of Ehrlichia are currently known to cause clinical and subclinical infections in dogs, i.e., E. canis, $E$. platys, E. equi, and E. risticii [10]. The illness caused by $E$. platys is commonly referred to as CICT, and was first described in the United States (U.S.A.) [5]. Dogs with CICT usually do not develop clinical illness, but show cyclic parasitemia of platelets followed by thrombocytopenia and general lymphadenomegaly. In the chronic stage of the disease, the cyclic nature of parasitized platelets sometimes diminishes, resulting in cyclic thrombocytopenia associated with irregularly occurring parasitemia [1,5].

Several effective drugs are available for the treatment of ehrlichial infections in dogs. Of these, tetracycline antibiotics (such as tetracycline, oxytetracycline, and doxycycline) are the most frequently used [6]. However, the duration of a given tetracycline treatment varies, and it is very difficult to determine the appropriate duration. The current recommendation is the administration of tetracycline at $22 \mathrm{mg} / \mathrm{kg}$ of body weight orally every $8 \mathrm{hr}$ for 14 to 21 days, or doxycycline at $10 \mathrm{mg} / \mathrm{kg}$ of body weight orally (or intravenously) every $24 \mathrm{hr}$ for 7 to 14 days $[6,10]$.

Investigations on the efficacies of tetracycline antibiotics against $E$. canis infection have been undertaken in the past, but it has been difficult to confirm if the infection has been eliminated or not by tetracycline treatments due to the nonavailability of appropriate techniques. Iqbal and Rikihisa have presented evidence in support of the carrier status of E. canis infection after doxycycline treatment [7]. In their study, the efficacy of doxycycline treatment in experimental E. canis infection was assessed by cell culture reisolation, immunofluorescence assay (IFA), and histopathologic

* Correspondence to: Dr. M.-J., Pan, Graduate Institute of Veterinary Medicine, College of Agriculture, National Taiwan University, 142 Chou-San Rd., Taipei, Taiwan 106, ROC. examination.

The efficacies of tetracycline for treatment of CICT have not been evaluated to date. In this communication, we demonstrate the ineffectiveness of tetracycline in completely eliminating $E$. platys infection in a dog. A healthy female crossbreed $\operatorname{dog} 6$ months of age $(\operatorname{dog} A)$ was inoculated with E. platys infected blood [1]. Venous blood samples were collected on alternate days with ethylene diaminetetraacetic acid as an anticoagulant. Automated platelet counts (Sysmex K-1000; Toa Medical Electronics Co., Ltd., Japan) and Giemsa staining were carried out, and the infected platelet ratio was estimated by observing 200 platelets in one blood smear. Tetracycline was then administered orally at $22 \mathrm{mg} / \mathrm{kg}$ of body weight 3 times a day for 2 weeks after the day of the parasitemic episode. On the sixth day of treatment, the platelet count rose and remained within the normal range (Fig. 1). After completion of the therapeutic regimen, the platelet count and blood smear staining were continued for 2 weeks. Although thrombocytopenia was no longer observed, a few E. platys soon reappeared sporadically in the platelets after the termination of treatment (Fig. 1).

Diagnosis of CICT could be performed either by demonstration of basophilic inclusions within the platelets in blood smears [5], by IFA [4], or by PCR [3]. Considering the fact that the parasitized platelets are cyclic in appearance and might be present only intermittently in the chronic stage, the determination of drug withdrawal by blood smear examination is not effective or reliable. Currently, the cultivation of E. platys has not been achieved, so the reisolation of etiologic agent as an indicator of therapeutic efficacy is not yet possible. Detection of antibody titer against $E$. platys by IFA is not good enough to conclude the time at which the antibiotic therapy may be withdrawn, since IFA is not able to assess the antibiotic efficacy until 2 months after the treatment when the specific antibody titer has decreased [7]. 


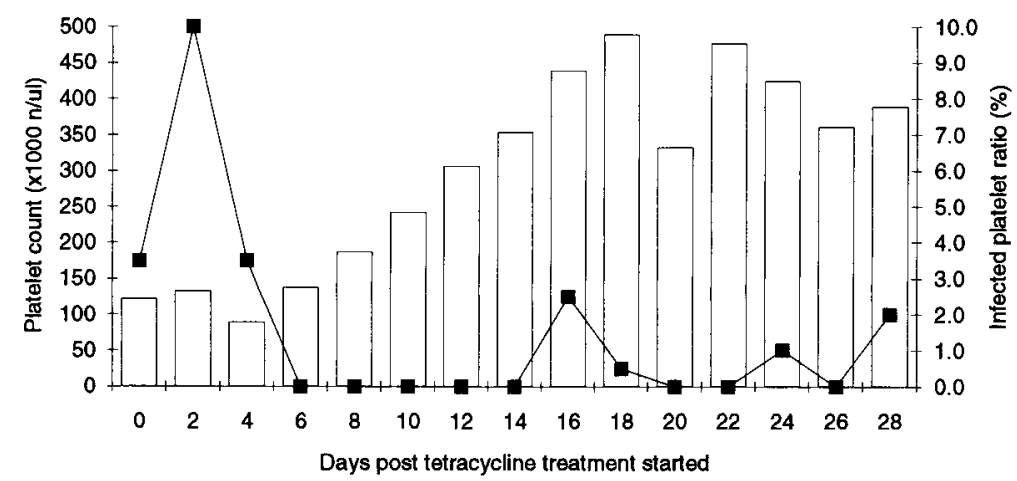

Fig. 1. Proportion of infected platelets and the platelet counts of dog A. Blood samples were collected on alternate days for 4 weeks post antibiotic treatment as the numbers indicate. Tetracycline was administered from day 1 to day 14 for 2 weeks. The number of platelets (bar area) is on the left axis, and the infected platelet ratio (solid line) is on the right axis.

We have developed a two-step PCR technique to specifically amplify the $16 \mathrm{~S}$ rDNA from $E$. platys [3]. The method is more sensitive and specific than the traditional diagnostic methods, and is practicable for diagnosis of CICT at both the early and late phases of infection [2,3]. We have also evaluated the feasibility of this technique in combination with Giemsa staining as a basis to determine the withdrawal time of treatment. Two 6-month-old male crossbreed $\operatorname{dogs}(\operatorname{dog} \mathrm{B}$ and $\operatorname{dog} \mathrm{C})$ in a healthy condition were experimentally infected with E. platys [1], and hematologic evaluations were carried out on alternate days. Each dog was administered doxycycline orally at $10 \mathrm{mg} / \mathrm{kg}$ of body weight daily when one parasitemic episode developed. Aliquots of each blood sample $(50 \mu l)$ were also assayed by the two-step PCR. In $\operatorname{dog}$ B, thrombocytopenia was cured after 6 days of doxycycline treatment (Fig. 2a). Both Giemsa staining and two-step PCR failed to detect $E$. platys on the sixth day post-treatment (Fig. 2). In $\operatorname{dog} \mathrm{C}$, both Giemsa staining and PCR could not detect the etiologic agent in the blood sample on the eighth day post-treatment (data not shown). Thus, the therapeutic regimens in 2 dogs were discontinued after 8 days of doxycycline administration. The monitoring continued, however, no E. platys was detected either by Giemsa staining or by the two-step PCR during the following 3 weeks (Fig. 2).

The dogs infected with E. platys do not become ill and rarely show signs of significant hemorrhage [1,5]; however, some reports suggest that more virulent strains may exist in nature $[8,9]$. In the treatment of ehrlichial diseases, the duration of therapy is more important than the dosage or frequency of drug administration [10]. The appropriate withdrawal time for ehrlichial infection depends on both the kind of antibiotics administered and the stage of the illness. Therefore, the duration of antibiotic treatment for CICT varies case by case, especially in the chronic stage, which often requires a longer therapeutic regimen. As our results show, the evaluation of therapeutic response by platelet counts alone would be misleading (Fig. 1), and we need more evidences to decide when to stop the treatment. The results of the present experiment indicate that the PCR amplification in combination with Giemsa staining is helpful in assessing the efficacy of antibiotic treatment for CICT as long as a reisolation technique for E. platys is not available.

\section{REFERENCES}

1. Chang, A. C.-H., Chang, W.-L., Lin, C.-T., Pan, M.-J., and Lee, S.-C. 1996. J. Vet. Med. Sci. 58: 473-476.

2. Chang, W.-L. and Pan, M.-J. 1996. J. Chin. Soc. Vet. Sci. 22: 9-16.

3. Chang, W.-L. and Pan, M.-J. 1996. J. Clin. Microbiol. 34: 3142-3146.

4. French, T. W. and Harvey, J. W. 1983. Am. J. Vet. Res. 44: 2407-2411.

5. Harvey, J. W., Simpso, C. F., and Gaskin, J. M. 1978. J. Infect. Dis. 137: 182-188.

6. Hoskins, J. D. 1991. Canine Pract. 16: 13-21.

7. Iqbal, Z. and Rikihisa, Y. 1994. J. Clin. Microbiol. 32: 1644 1649.

8. Kontos, V. J., Papadopoulos, O., and French, T. W. 1988. 4th Hellenic Vet. Congr. Abstr. 113-114.

9. Wilson, B. J. 1992. J. Am. Anim. Hosp. Assoc. 28: 381-383.

10. Woody, B. J. and Hoskins, J. D. 1991. Vet. Clin. North Am. Small Anim. Pract. 21: 75-98. 


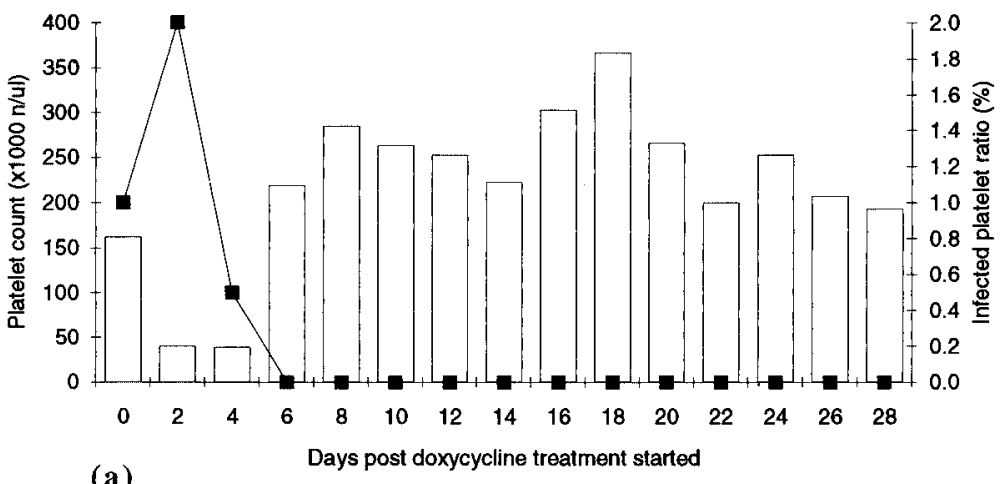

(a)

Days post doxycycline treatment started

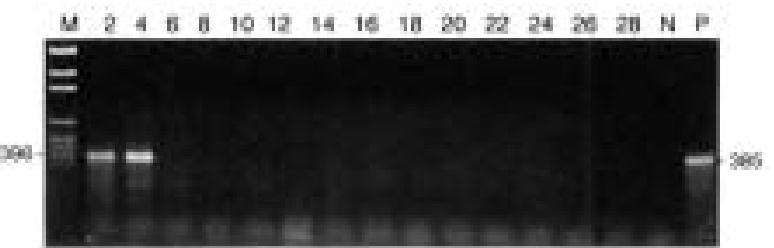

(b)

Fig. 2. Follow-up monitors of dog B during the duration of doxycyline treatment and withdrawal. Blood specimens were collected on alternate days for 4 weeks as the numbers indicate, and doxycycline was administered from day 1 to day 8. (a) Proportion of platelets infected with E. platys and platelet counts. The number of platelets (bar area) is on the left axis, and the infected platelet ratio (solid line) is on the right axis. (b) Agarose gel electrophoresis of the second-step PCR-amplified products. Lane M, molecular size standards (pGEM DNA marker; Promega, U.S.A.); lane N, no DNA control; lane P, positive control with E. platys genomic DNA as a template. The approximate size of PCR products is marked on the right of the gel. 